\title{
High Sensitivity Plasmonic Metal-Dielectric-Metal Device With Two Side-Coupled Fano Cavities
}

\author{
Yunqing LU*, Jiong XU, Min XU, Ji XU, Jin WANG, and Jiajin ZHENG \\ College of Electronic and Optical Engineering \& College of Microelectronics, Nanjing University of Posts and \\ Telecommunications, Nanjing 210023, China \\ "Corresponding author: Yunqing LUＥ-mail: luyq@njupt.edu.cn
}

\begin{abstract}
In this paper, we propose a compact plasmonic sensor structure comprised of a metal-dielectric-metal (MDM) waveguide, and a baffle plate in waveguide core and two side-coupled rectangular cavities. In this structure, two Fano resonances are achieved and can be tuned independently by changing the structural parameters of the cavities. Especially, when the resonant wavelengths of the two Fano resonances are the same, the sensing sensitivity can be enhanced by coupling between two Fano resonances. By investigating the transmission spectrum, the effect of structural parameters on Fano resonances and the refractive index sensitivity of the sensor structure are analyzed in detail. The numerical simulations demonstrate a sensitivity as high as $1295 \mathrm{~nm} / \mathrm{RIU}$ and a figure of merit of 1647.
\end{abstract}

Keywords: Fano resonance; MDM waveguide; sensor

Citation: Yunqing LU, Jiong XU, Min XU, Ji XU, Jin WANG and Jiajin ZHENG, "High Sensitivity Plasmonic Metal-Dielectric-Metal Device With Two Side-Coupled Fano Cavities," Photonic Sensors, 2019, 9(3): 205-212.

\section{Introduction}

Surface plasmon polaritons (SPPs) are surface electromagnetic waves propagating along a metal-dielectric interface and have great potential applications in the realization of highly integrated optical circuits due to their capability to overcome the diffraction limit of light [1]. Nanoplasmonic waveguides, supporting the propagation of SPPs and manipulating light on a subwavelength scale, can be regarded as an ideal optical element in an integrated plasmonic device. Among a variety of plasmonic waveguide structures, the metal-dielectric-metal (MDM) waveguide is one of the most promising structures in highly integrated photonic nano-devices due to the qualities of deep-subwavelength field confinements, low bend loss, long propagation lengths, and easy fabrication [2]. Various waveguide-coupled resonator devices based on the MDM waveguide have been proposed and demonstrated, such as filters [3], switches [4], and sensors [5]. Among these devices, plasmonic sensors for refractive index sensing have attracted intense interest due to their promising applications in the biological and chemical detection [6]. The principle of resonator-type refractive index sensors is based on the strong dependence of the resonance frequency on the environment. High refractive-index sensitivity and high figure of merit (FOM) are pursued in the design of plasmonic sensors, which depend on spectral shapes. Higher sensitivity and FOM can be achieved by sharper spectral shape.

Fano resonances are quite preferred in the ultra-sensitive sensors [8], and especially,

Received: 30 November 2018 / Revised: 2 April 2019

(C) The Author(s) 2019. This article is published with open access at Springerlink.com DOI: $10.1007 / \mathrm{s} 13320-019-0555-8$

Article type: Regular 
ultracompact plasmonic sensors with high sensitivity can be realized by combining the Fano resonance with MDM plasmonic structures. As demonstrated in quantum systems, Fano resonance occurs due to the interference between a discrete energy state and a continuum spectrum of an elementary excitation, and possesses a distinctly asymmetric line profile [7]. The spectral lines of Fano resonance are much narrower as compared with the regular Lorentzian line shape. Thus, the plasmonic refractive index sensors based on various MDM waveguide coupled resonator systems with Fano resonance have been proposed and investigated [9-14]. Wen et al. proposed an MDM-waveguide structure coupled with an end-coupled ring groove [9]. Qiao et al. proposed an MDM-waveguide structure coupled with an M-type cavity [10]. Yun et al. designed a stub and circular cavity coupled structure [11]. Recently, dual and multiple Fano resonances in a single MDM structure become more important and have gained much attention to enhance bio-chemical sensing and develop high-integrated photonic circuit. For example, dual Fano resonances are achieved in an MDM waveguide with a slot cavity below or above a groove with a sensitivity of $1131 \mathrm{~nm} / \mathrm{RIU}$ [15], and an MDM waveguide with double tooth-shaped cavities with a sensitivity of 1000 nm/RIU [16]. Multiple Fano resonances are achieved in an MDM waveguide coupled with a disk with a sensitivity of $1100 \mathrm{~nm} / \mathrm{RIU}$ [17], an MDM waveguide structure composed of several tangent-ring resonators with a sensitivity of $880 \mathrm{~nm} / \mathrm{RIU}$ [18], and by employing an end-coupled slot cavity resonator with a sensitivity of $950 \mathrm{~nm} / \mathrm{RIU}$ [19]. However, the coupling between different Fano resonances is rarely emphasized and the highest sensitivity is $1131 \mathrm{~nm} / \mathrm{RIU}$ among those structures. Moreover, the tunability of the Fano resonances is desired for many applications.

In this paper, two Fano resonances are constructed in a compact plasmonic system, which consists of an MDM waveguide, a baffle plate in waveguide core, and two side-coupled rectangular cavities. These two side-coupled rectangular cavities provide two discrete states, while the baffle plate in the waveguide core produces a continuous transmission spectrum. In fact, two discrete states coupled with the continua will lead to two Fano resonances. The effect of structural parameters on the Fano resonances and the tunability of the Fano resonances are analyzed. The sensing sensitivity can be improved by coupling between the two Fano resonances, when the resonant wavelengths of the two Fano resonances are the same.

\section{Structure model and theoretical analysis}

The detailed structure of the proposed side-coupled resonator system is schematically shown in Fig. 1. The waveguide core with a width of $w$ is sandwiched by a semi-infinite metal claddings. A baffle plate with a thickness of $d_{\mathrm{m}}$ is placed in the waveguide core. Two rectangular cavities A and B are placed on both sides of the bus waveguide. The height and width of the rectangular cavity A are $H_{1}$ and $L_{1}$, and the coupling distance between cavity $\mathrm{A}$ and bus waveguide is $G_{1}$. The height and width of the rectangular cavity B are $H_{2}$ and $L_{2}$, respectively, and the coupling distance between cavity $\mathrm{B}$ and bus waveguide is $G_{2}$. The metal is chosen as silver whose permittivity can be expressed by the Drude model [20]:

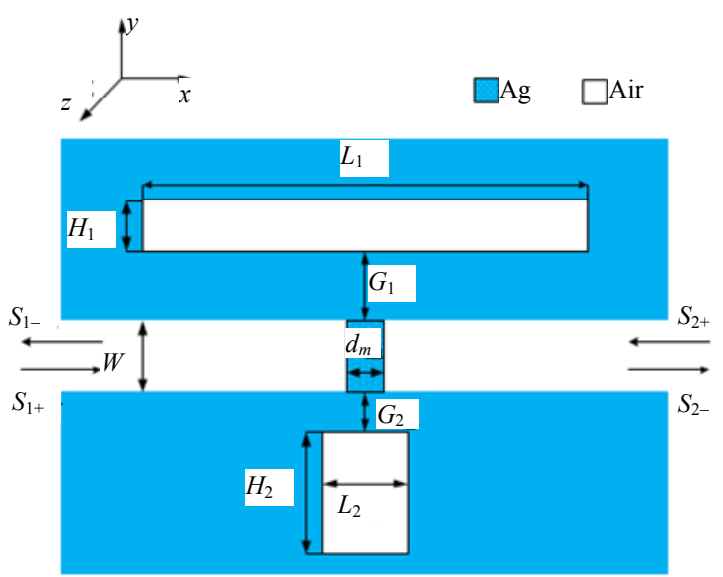

Fig. 1 Schematic diagram of the side-coupled MDM waveguide structure. 


$$
\varepsilon_{m}(\omega)=\varepsilon_{\infty}-\frac{\omega_{p}^{2}}{\omega^{2}+\mathrm{i} \omega \gamma}
$$

where $\varepsilon_{\infty}=3.7, \omega_{p}=9 \mathrm{eV}$, and $\gamma=0.018 \mathrm{eV}$ represent the interband-transition contribution to the permittivity, the bulk plasma frequency, and the electron collision frequency, respectively.

In the MDM structure, two Fano resonances arise from the coherent coupling and interference between a discrete state and a continuous state as follows. When a TM-polarized light is launched from the left side to the MDM waveguide, the fundamental TM mode, i.e. the SPP mode, will be excited and propagate along the waveguide. If there is no baffle plate in the waveguide core, the SPPs will be coupled into the rectangular cavities with special wavelengths, and the transmission spectrum exhibit the Lorentzian-like line shape. With the baffle plate, SPPs go to the surface of the baffle plate and are reflected back, which produces a continuous transmission spectrum. An asymmetric Fano line shape will be achieved by the interference between the discrete state and continuous state provided by the rectangular cavities and the baffle plate, respectively.

To reveal the origin of the Fano resonances, the transmission of this structure is analyzed by utilizing the temporal coupled mode theory [21-23]. First, only rectangular cavity A is considered. For characterizing the cavity, we define the following parameters: $\omega_{0}$ is the resonance frequency, $a$ is the cavity mode amplitude, and $d_{1}$ and $d_{2}$ are the input coupling coefficients of the forward and backward propagating modes in the waveguide. $s_{i^{+}}$and $s_{i-}$ $(i=1,2)$ are the amplitudes of the incoming and outgoing waves into the waveguide, respectively. $1 / \tau_{A}$ is the intrinsic cavity loss, and $1 / \tau_{1}$ and $1 / \tau_{2}$ are the decay rates of the cavity amplitude into the two ports, respectively. $Q_{A}$ is the quality factor due to the intrinsic loss in the cavity, and $Q_{e}$ is the quality factor related to waveguide coupling loss. The decay rates are related to the cavity quality factors by $Q_{A}=\left(\omega_{0} \tau_{A}\right) / 2$ and $Q_{e}=\omega_{0}\left(\tau_{1}+\tau_{2}\right) / 4$. The time evolution of the amplitude of rectangular cavity $\mathrm{A}$ in the steady state can be described as

$$
\frac{d a}{d t}=\left(-\mathrm{j} \omega_{0}-\frac{2}{\tau_{A}}-\frac{2}{\tau_{1}}-\frac{2}{\tau_{2}}\right) a+d_{1} s_{1+}+d_{2} s_{2+} .
$$

By power conservation, the outgoing waves are

$$
\left(\begin{array}{l}
s_{1-} \\
s_{2-}
\end{array}\right)=\mathbf{C}\left(\begin{array}{l}
s_{1+} \\
s_{2+}
\end{array}\right)+\left(\begin{array}{l}
d_{1} \\
d_{2}
\end{array}\right) a .
$$

The scattering matrix $\mathbf{C}$ represents the direct coupling between the incoming and outgoing waves, expressed as

$$
\mathbf{C}=\left(\begin{array}{c}
r_{B}-\mathrm{j} t_{B} \\
-\mathrm{j} t_{B} r_{B}
\end{array}\right)
$$

where $r_{B}$ and $t_{B}$ are the corresponding amplitude reflection coefficient and transmission coefficient when the SPPs pass through the baffle and satisfy $r_{B}^{2}+t_{B}^{2}=1$. Considering the symmetry of our structure, we have $\tau_{1}=\tau_{2}=\tau$. So the transfer function of the system can be expressed as

$$
t(\omega)=-\mathrm{j} t_{B}-\frac{4 / \tau\left(r_{B}-\mathrm{j} t_{B}\right)}{\mathrm{j}\left(\omega_{0}-\omega\right)+2 / \tau_{A}+4 / \tau}
$$

and the transmission spectrum is

$$
T(\varpi)=\left|t_{B}+\mathrm{j} t_{B} r_{B}-\frac{1 / Q_{e}}{2 \mathrm{j}\left(1-\omega / \omega_{0}\right)+1 / Q_{A}+1 / Q_{e}}\right|^{2} .
$$

From (6), it can be seen that the transmittance is mainly dependent on the cavity quality factors and reflectivity amplitude $r_{B}$. Figure 2(a) shows the transmission spectra as a function of wavelength for different reflectivity amplitudes, where $Q_{A}=300$ and $Q_{e}=100$. When there is no baffle plate existing $\left(r_{B}=0\right)$, the transmission spectrum exhibits Lorentzian profile with a resonant wavelength of $1312 \mathrm{~nm}$. When $r_{B} \neq 0$, the input SPPs waves are divided into two parts: one part is coupled to cavity and results in a discrete state, and the other part directly passes through the baffle plate and gives rise to a broad state. The interference between these two states leads to the sharp and asymmetric Fano resonance. These spectra lines show a typical Fano resonance like feature. The strength of the Fano resonance depends on the reflectivity amplitude. The 
resonance peak becomes narrower with an increase in the reflectivity amplitude. Figure 2(b) shows the transmission spectra as a function of wavelength for different values of quality factor $Q_{e}$, where $Q_{A}=300$ and $r_{B}=0.95 . Q_{e}$ is the quality factor related to waveguide coupling loss, which depends on the coupling distance between cavity A and waveguide $G_{1}$. The waveguide coupling loss increases with an increase in $Q_{e}$, and the strength of the Fano resonance decreases gradually.

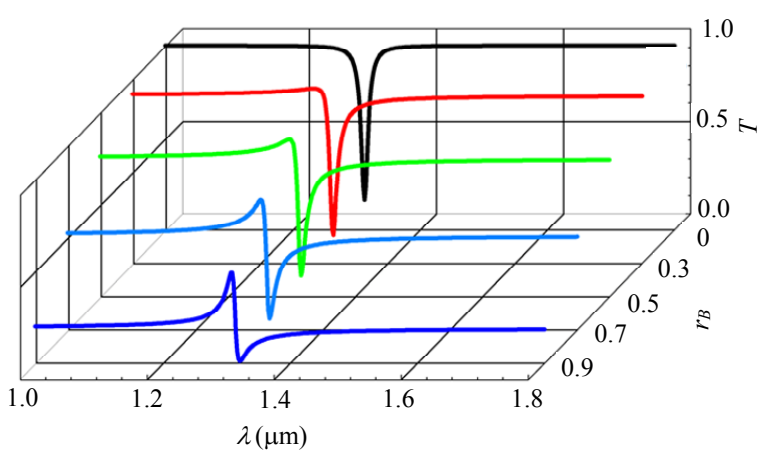

(a)

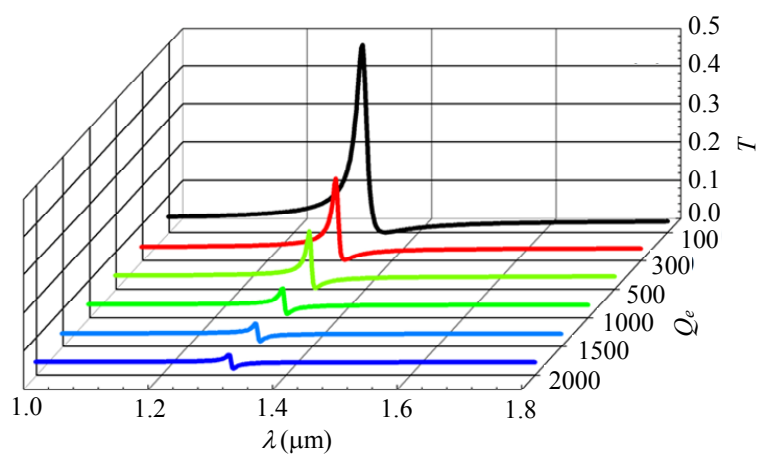

(b)

Fig. 2 Evolution of transmission spectra of the proposed structure: (a) transmission spectra of the proposed structure as a function of wavelength for different reflectivity amplitude, where $Q_{A}=300$ and $Q_{e}=100$ and (b) transmission spectra of the proposed structure as a function of wavelength for different value of quality factor $Q_{e}$, where $Q_{A}=300$ and $r_{B}=0.95$.

Then, both rectangular cavities $\mathrm{A}$ and $\mathrm{B}$ are considered. The resonant frequencies for rectangular cavities $\mathrm{A}$ and $\mathrm{B}$ are $\omega_{A}$ and $\omega_{B}$. The two rectangular cavities couple with each other by a coupling coefficient $\mu$. The intrinsic loss of rectangular cavity $\mathrm{B}$ is $1 / \tau_{B}$. The CMT equations can be expressed as

$$
\frac{d a}{d t}=\left(-\mathrm{j} \omega_{A}-\frac{2}{\tau_{A}}-\frac{2}{\tau_{1}}-\frac{2}{\tau_{2}}\right) a+d_{1} s_{1+}+d_{2} s_{2+}-\mathrm{j} \mu b
$$

The transfer function can be expressed as

$$
\begin{aligned}
& t(\omega)=-\mathrm{j} t_{B}- \\
& 2\left[\mathrm{j}\left(\omega_{B}-\omega\right)+2 / \tau_{B}\right]\left(r_{B}-\mathrm{j} t_{B}\right) / \tau \\
& \frac{\left.\mathrm{j}\left(\omega_{A}-\omega\right)+2 / \tau_{A}+4 / \tau\right]\left[\mathrm{j}\left(\omega_{B}-\omega\right)+2 / \tau_{B}\right]+\mu^{2}}{} .
\end{aligned}
$$

\section{Results and discussion}

To realize the above results of the theoretical model, the transmission spectra and filed distributions of the proposed structure are investigated with the finite element method (FEM). In our simulations, the structure parameters are chosen as: $w=50 \mathrm{~nm}, L_{1}=400 \mathrm{~nm}, H_{1}=50 \mathrm{~nm}, G_{1}=$ $10 \mathrm{~nm}, L_{2}=50 \mathrm{~nm}, H_{2}=430 \mathrm{~nm}, G_{2}=10 \mathrm{~nm}$, and $d_{m}=$ $20 \mathrm{~nm}$. The transmission spectrum of the rectangular cavity A coupled with the MDM waveguide is presented in Fig. 3(a). When only baffle plate is placed in the waveguide core, a continuous transmission spectrum with low transmittance is produced, as shown by the dashed line. When only rectangular cavity A is considered, the transmission spectrum of the rectangular cavity coupled with the MDM waveguide is presented by the solid line. There is a symmetric Lorentzian-like valley, which represents the eigenmode of the side-coupled resonator. A discrete state is provided by the side-coupled rectangular cavity. The interference between the discrete state and continuous state leads to the Fano resonance, as shown by the dotted line. A resonance peak with asymmetric line shape is observed. The slope of the left shoulder of the resonance peak is obviously larger than that of the right shoulder, which is a typical Fano profile with one maximum $(1299 \mathrm{~nm})$ and one minimum $(1250 \mathrm{~nm})$. The transmittance is near to 0 at the transmission dip. To further understand the formation mechanism of the Fano resonances, the field distributions of $\left|H_{z}\right|$ at the transmitted peak and 
dip are displayed in Figs.3(b) and 3(c). It is evident that the Fano resonance peak corresponds to standing wave pattern. The Fano resonance peak can be tuned by changing the refractive index of the medium in the rectangular cavity, which means it can be applied as a refractive index sensor. For the Fano resonances of the proposed side-coupled system, the extremely steep change from resonant peak to dip indicates a highly sensitive response to the refractive index variation of the dielectric environment. In refractive index sensing applications, sensitivity $(S)$ is usually used to evaluate the sensing performance, which is defined as $S=\Delta \lambda / \Delta n$, where $\Delta n$ is the refractive index change and $\Delta \lambda$ is the resonance wavelength change owing to the refractive index change. The wavelength of the Fano resonance peak as a function of the refractive index is shown in Fig. 3(d). The refractive index $n$ increases from 1.00 to 1.20 with an interval of 0.05 . The sensitivity $S$ of the side-coupled structure is $1245 \mathrm{~nm} / \mathrm{RIU}$.
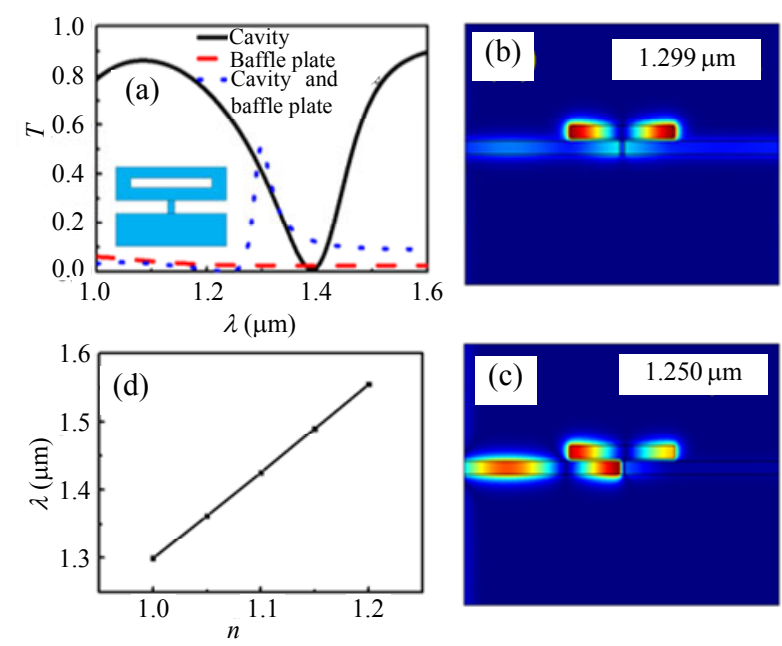

Fig. 3 Simulation results, where only rectangular cavity A is considered: (a) transmission spectra of the side-coupled resonator systems, (b) and (c) the field distributions of $\left|H_{z}\right|$ at the transmitted peak $(1299 \mathrm{~nm})$ and dip $(1250 \mathrm{~nm})$, respectively, and (d) the wavelength of the Fano resonance peak as a function of the refractive index.

When only rectangular cavity $\mathrm{B}$ is considered, the transmission spectrum of the rectangular cavity coupled with the MDM waveguide is shown in
Fig. 4(a). A Fano resonance also can be observed, which arises from the interference between the discrete state provided by the side-coupled rectangular cavity $\mathrm{B}$ and continuous state provided by the baffle plate. Compared with Fig. 3(a), full wave at half maximum (FWHM) of the resonance peak is narrower. Figures 4 (b) and 4(c) show the field distributions of $\left|H_{z}\right|$ at the transmitted peak $(1312 \mathrm{~nm})$ and dip $(1350 \mathrm{~nm})$, respectively. The wavelength of the Fano resonance peak as a function of the refractive index is shown in Fig.4(d). Due to the narrower FWHM, the sensitivity $S$ of the sensor increases from $1245 \mathrm{~nm} / \mathrm{RIU}$ to $1280 \mathrm{~nm} / \mathrm{RIU}$.
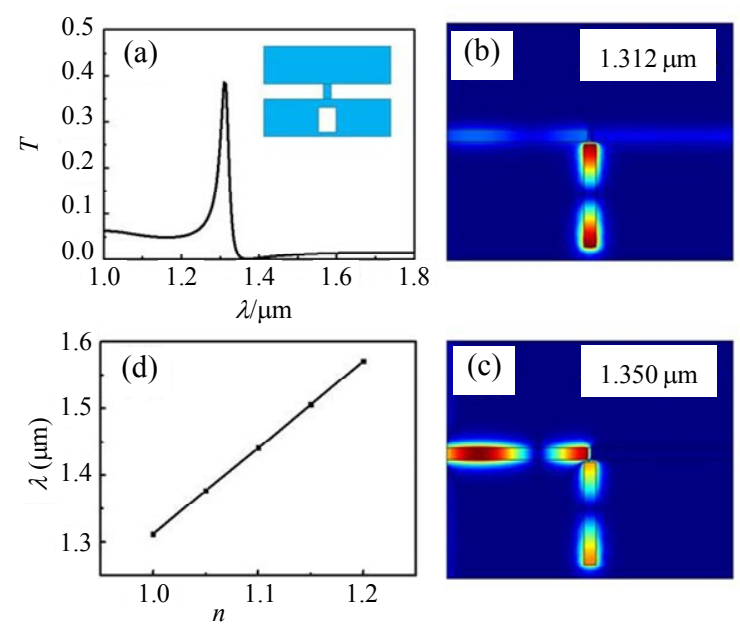

Fig. 4 Simulation results, where only rectangular cavity B is considered: (a) transmission spectra of the side-coupled resonator systems, (b) and (c) the field distributions of $\left|H_{z}\right|$ at the transmitted peak $(1312 \mathrm{~nm})$ and dip $(1350 \mathrm{~nm})$, respectively, and (d) the wavelength of the Fano resonance peak as a function of the refractive index.

When both rectangular cavities $\mathrm{A}$ and $\mathrm{B}$ are considered, two discrete states are provided. Two Fano resonances are achieved by the coupling between the two discrete states and the continuum state, as shown in Fig. 5(a). These two Fano resonance peaks can be tuned independently by changing the parameters of the rectangular cavities. The transmission spectra of the proposed system for $L_{1}=0.48 \mu \mathrm{m}, 0.46 \mu \mathrm{m}, 0.44 \mu \mathrm{m}, 0.42 \mu \mathrm{m}$, and $0.4 \mu \mathrm{m}$ are depicted in Figs. 5(a)-5(e), respectively. The second resonance peak is blue-shifted with a 
decrease in the width $L_{1}$ of the rectangular cavity A. But since $L_{2}$ has the same value, the left Fano peak is always at the same wavelength. Therefore, by changing the structural parameters of the cavities appropriately, it is easy to design a specific Fano peak at the desired wavelength without any impact on another peak. This feature provides great flexibility in the design of the sensor. When the resonant wavelengths of the two Fano resonances are the same, the sensing sensitivity can be further improved by coupling between the two Fano resonances. The transmission spectra of the side-coupled resonator system with different dielectric refractive indexes of $n$ are shown in Fig. 6(a), and the wavelength of the Fano resonance peak as a function of the refractive index is shown in Fig. 6(b). The sensitivity $S$ of the sensor increases to $1295 \mathrm{~nm} / \mathrm{RIU}$.

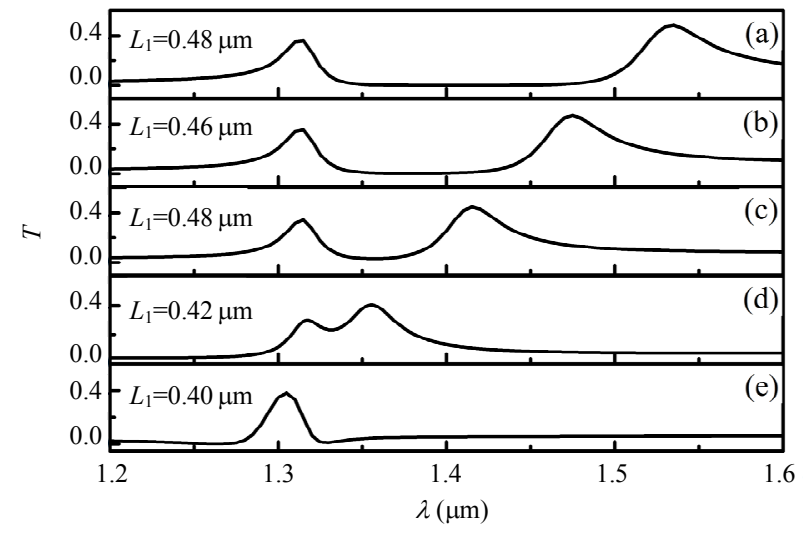

Fig. 5 Transmission spectra of the side-coupled resonator systems with (a)-(e): $L_{1}=0.48 \mu \mathrm{m}, L_{1}=0.46 \mu \mathrm{m}, L_{1}=0.44 \mu \mathrm{m}$, $L_{1}=0.42 \mu \mathrm{m}$, and $L_{1}=0.4 \mu \mathrm{m}$, respectively.

Additionally, FOM as a key factor is widely used to evaluate the performance of the Fano resonance. A high FOM value will be preferred in Fano resonance for the sensing applications, and then $F O M^{*}$ and $F O M$ are defined as

$$
\begin{gathered}
F O M^{*}=\left|\frac{d T(\lambda) / d n(\lambda)}{T(\lambda)}\right| \\
F O M=\max \left(F O M^{*}\right)
\end{gathered}
$$

where $T(\lambda)$ is the transmittance at the specific wavelength, and $d T(\lambda) / d n(\lambda)$ is the transmittance change at the fixed wavelength induced by a refractive index change. According to (10) and (11), a relative transmittance change at fixed wavelength $\lambda$ can be detected for the Fano resonance. It can be predicted that an ultra-low transmittance and a sharp change of the transmittance induced by the index changes are preferred for obtaining a large FOM. Based on the transmission spectra in Figs.3(a), 4(a), and Fig. 6(a), the calculated values of $F^{*}{ }^{*}$ at different wavelengths by changing the refractive index from 1 to 1.2 are shown in Fig. 7. For the case of only considering cavity A, only considering cavity $\mathrm{B}$ and considering both cavity $\mathrm{A}$ and $\mathrm{B}$, the values of FOM are 222, 1072, and 1647, respectively. It is proved that the changes of the transmittance induced by the index changes become sharper through the coupling between the two Fano resonances, and the FOM is improved significantly.

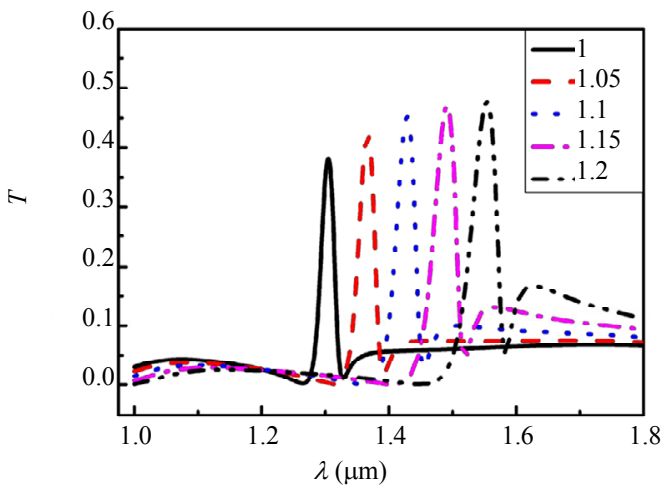

(a)

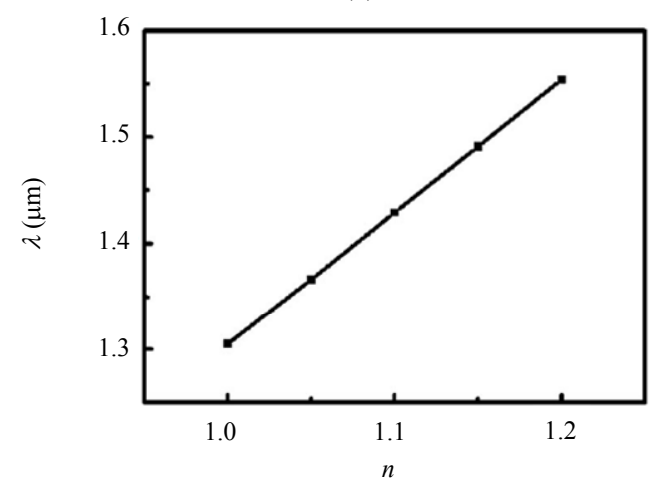

(b)

Fig. 6 Transmission spectra of the side-coupled resonator system with (a) different dielectric refractive indexes $n$ and (b) resonance wavelength as a function of the refractive index. 


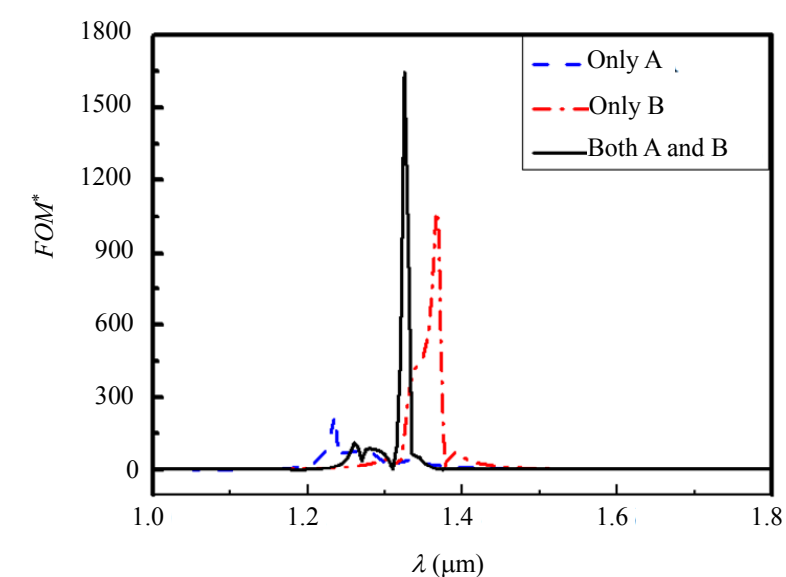

Fig. 7 Calculated $F^{*}$ at different wavelengths for rectangular cavity A (blue dashed line) and rectangular cavity B (red dotted line), and both rectangular cavities A and B are considered (black solid line).

\section{Conclusions}

We present two Fano resonances in a side-coupled resonator system, which consists of a MDM waveguide coupled with two side rectangular cavities and a baffle plate in waveguide core. The two Fano resonances result from the interference between two discrete states and a continuum state. These two Fano resonances can be tuned independently by changing the parameters of the rectangular cavities. The sensing applications of the proposed system are discussed, and a high sensitivity of $1295 \mathrm{~nm} / \mathrm{RIU}$ is achieved. It is believed that the proposed structure can be applied in the on-chip optical sensing

\section{Acknowledgment}

This work was supported by the National Natural Science Foundation of China (Grant Nos. 61575096 and 11404170); the Scientific Research Project of Nanjing University of Posts and Telecommunications (Grant No. NY217110).

Open Access This article is distributed under the terms of the Creative Commons Attribution 4.0 International License (http://creativecommons.org/licenses/by/4.0/), which permits unrestricted use, distribution, and reproduction in any medium, provided you give appropriate credit to the original author(s) and the source, provide a link to the Creative Commons license, and indicate if changes were made.

\section{References}

[1] J. M. Pitarke, V. M. Silkin, E. V. Chulkov, and P. M. Echenique, "Theory of surface plasmons and surface-plasmon polaritons," Reports on Progress in Physics, 2006, 70(1): 1-87.

[2] J. A. Dionne, L. A. Sweatlock, H. A. Atwater and A. Polman, "Plasmon slot waveguides: towards chip-scale propagation with subwavelength-scale localization," Physical Review B, 2006, 73(3): 035407-1-035407-9.

[3] W. Lai, K. Wen, J. Lin, Z. Guo, Q. Hu, and Y. Fang, "Plasmonic filter and sensor based on a subwavelength end-coupled hexagonal resonator," Applied Optics, 2018, 57(22): 6369-6374.

[4] M. Fang, F. Shi, and Y. Chen, "Unidirectional all-optical absorption switch based on optical Tamm state in nonlinear plasmonic waveguide," Plasmonics, 2016, 11: 197-203.

[5] H. Lu, X. Liu, D. Mao, and G. Wang, "Plasmonic nanosensor based on Fano resonance in waveguidecoupled resonators," Optics Letters, 2012, 37(18): 3780-3782.

[6] X. Zhou, L. Zhang, M. Armani, D. Zhang, X. Duan, J. Liu, et al., "On-chip biological and chemical sensing with reversed Fano lineshape enabled by embedded microring resonators," IEEE Journal of Selected Topics in Quantum Electronics, 2014, 20(3): 35-44.

[7] U. Fano, "Effects of configuration interaction on intensities and phase shifts," Physical Review, 1961, 124(6): 1866-1878.

[8] Z. Chen, Y. Li, L. Wang, G. Duan, Y. Zhao, and J. Xiao, "Sharp asymmetric line shapes in a plasmonic waveguide system and its application in nanosensor," Journal of Lightwave Technology, 2015, 33(15): 3250-3253.

[9] K. Wen, Y. Hu, L. Chen, J. Zhou, M. He, L. Lei, et al., "Fano resonance based on end-coupled cascaded-ring MIM waveguides structure," Plasmonics, 2017, 12(6): 1875-1880.

[10] L. Qiao, G. Zhang, Z. Wang, G. Fan, and Y. Yan, "Study on the Fano resonance of coupling M-type cavity based on surface plasmon polaritons," Optics Communications, 2019, 433: 144-149.

[11] B. Yun, R. Zhang, G. Hu, and Y. Cui, "Ultra sharp Fano resonances induced by coupling between plasmonic stub and circular cavity resonators," Plasmonics, 2016 11(4): 1157-1162.

[12] H. Lu, X. Liu, and D. Mao, "Plasmonic analog of electromagnetically induced transparency in multi-nanoresonator-coupled waveguide systems," Physical Review A, 2012, 85(5): 53803-1-53803-7.

[13] Y. Deng, G. Cao, H. Yang, G. Li, X. Chen, and W. Lu, "Tunable and high-sensitivity sensing based on Fano resonance with coupled plasmonic cavities," Scientific Reports, 2017, 7(1): 10639.

[14] S. Zhan, H. Li, G. Cao, Z. He, B. Li, and H. Yang, 
"Slow light based on plasmon-induced transparency in dual-ring resonator-coupled MDM waveguide system," Journal of Physics D: Applied Physics, 2014, 47(20): 205101.

[15] K. Wen, Y. Hu, L. Chen, J. Zhou, L. Lei, and Z. Meng, "Single/dual Fano resonance based on plasmonic metal-dielectric-metal waveguide," Plasmonics, 2016, 11: 315-321.

[16] X. Shi, L. Ma, Z. Zhang, Y. Tang, Y. Zhang, J. Han, et al., "Dual Fano resonance control and refractive index sensors based on a plasmonic waveguide-coupled resonator system," Optics Communications, 2018, 427: 326-330.

[17] C. Li, S. Li, Y. Wang, R. Jiao, L. Wang, and L. Yu, "Multiple Fano resonances based on plasmonic resonator system with end-coupled cavities for high performance nanosensor," IEEE Photonics Journal, 2017, 9(6): 4801509.

[18] Z. Guo, K. Wen, Q. Hu, W. Lai, J. Lin, and Y. Fang, "Plasmonic multichannel refractive index sensor based on subwavelength tangent-ring metal- insulator-metal waveguide," Sensors, 2018, 18(5): 1348.

[19] K. Wen, L. Chen, J. Zhou, L. Lei, and Y. Fang, “A plasmonic chip-scale refractive index sensor design based on multiple Fano resonances," Sensors, 2018, 18(10): 3181.

[20] P. B. Johnson and R. W. Christy, "Optical constants of the Noble metals," Physical Review B, 1972, 6(12): 4370-4379.

[21] W. Suh, Z. Wang, and S. Fan, "Temporal coupledmode theory and the presence of non-orthogonal modes in lossless multimode cavities," IEEE Journal of Quantum Electronics, 2004, 40(10): 1511-1518.

[22] S. Fan, W. Suh, and J. D. Joannopoulos, “Temporal coupled-mode theory for the Fano resonance in optical resonators," Journal of Optical Society of America A, 2003, 20(3): 569-72.

[23] Q. Li, T. Wang, Y. Su, M. Yan, and M. Qiu, "Temporal coupled-mode theory for the Fano resonance in optical resonators," Optics Express, 2010, 18(8): 8367-8382. 